\title{
Open-Cell Aluminium Foams with Graded Coatings as Passively Controllable Energy Absorbers
}

\author{
A. Jung ${ }^{a, *}$, L.A.A. Beex ${ }^{b}$, S. Diebels ${ }^{a}$, S.P.A. Bordas ${ }^{b, c}$ \\ ${ }^{a}$ Saarland University, Lehrstuhl für Technische Mechanik \\ Campus A4.2, 66123 Saarbrucken, Germany \\ ${ }^{b}$ University of Luxembourg, Research Unit of Engineering Science, Campus Kirchberg \\ 6 rue Richard Coudenhove-Kalergi, L-1359 Luxembourg, Luxembourg \\ ${ }^{c}$ Cardiff University, School of Engineering, Queen's Buildings \\ The Parade, Cardiff CF24 3AA, Wales, UK
}

\begin{abstract}
Compared to most bulk materials, open-cell aluminium (Al) foams (OCAFs) are light-weight and can absorb a significant amount of energy in compression, e.g. during impact. When coated with nickel (Ni), OCAFs can absorb even more energy, making them more appropriate for impacts at higher velocities than uncoated OCAFs. When Ni-coated OCAFs experience low-velocity impact however, the stopping distance during the impact is small compared to that of uncoated OCAFs and hence, deceleration occurs fast. This exposes devices (and possibly human beings) protected by OCAFs to large internal forces leading to internal damage. An OCAF that combines the properties of uncoated and coated OCAFs can absorb energy during both low-velocity and high-velocity impact scenarios. This contribution introduces two of such OCAFs which are created by partially and gradually coating OCAFs. The general mechanics of the two OCAFs are revealed using experimental and numerical observation methods.
\end{abstract}

Keywords: Open-cell metal foams, Hybrid metal foams, Energy absorbing materials, Functionally graded materials, Coating, Impact

\footnotetext{
${ }^{*}$ Corresponding author

Email: anne.jung@mx.uni-saarland.de
} 


\section{Introduction}

Open-cell metal foams (OCMFs) can be used in a wide variety of engineering applications. They can amongst others be used as catalyst support [1, 2], implant material [3], heat exchanger [4], sound absorber [5] and energy/impact absorbers $[6,7,8,9,10,11,12,13]$. In the future OCMFs may have the potential to partially replace the relatively heavy bulk metals used in crumple zones of cars as low-weight energy absorbers [14].

The convenient energy-absorbing properties of OCMFs in compression can be distinguished in Fig. 1 (a), e.g. for the curve denoted by ' $50 \mu \mathrm{m}$ '. The compressive response initially shows an almost linear regime up to a strain of approximately half of the pore size, at which a stress peak is present. Since in this almost linear regime a small number of struts already deform plastically, it is often called the pseudo-elastic regime. Consequently, the Young's modulus of OCMFs is determined based on the unloading response. At a strain corresponding to the full pore size a stress plateau is reached. The plateau continues up to a densification strain, at which all pores are compressed. This wide stress plateau is beneficial for energy absorption, as the area under the curve is roughly the energy dissipated in compression, i.e. during impact.

OCMFs owe their wide stress plateau as well as their low weight to their distinct mesostructure, which consists of relatively slim, metal struts connected in a sparse manner (see Fig. 1 (b)). During the initial elastic stage of compression, most struts deform nearly elastically. The stress peak that ends the elastic stage, the so-called plastic collapse stress, is governed by the buckling and failure of one row of struts in the foam. During the stage of the stress plateau, the other rows of struts buckle and fail consecutively. When all struts have buckled and failed at the end of the compression, densification occurs and the stress grows significantly for small increases of strain [15].

OCMFs can be made of several metals and/or alloys (e.g. PtFeAlO, NiTi, Ta) $[1,3,16]$. This study focuses on those made of aluminium (Al) $[6,9$, $10,11,12,13,14,15]$. Jung et al. have shown that the energy-absorbing characteristics of OCAFs can be greatly enhanced by coating them with nickel $(\mathrm{Ni})[11,12,13,15]$. The Ni-coated foams are called Ni/Al hybrid foams. The process to apply the Ni-coating uses electrodeposition. OCAFs with Ni-coatings show substantially higher compressive stress-strain curves (see Fig. 1 (a)). As a result, the energy absorption is significantly improved. For a coating thickness of $150 \mu \mathrm{m}$, the absolute energy-absorbing capacity 
(a)

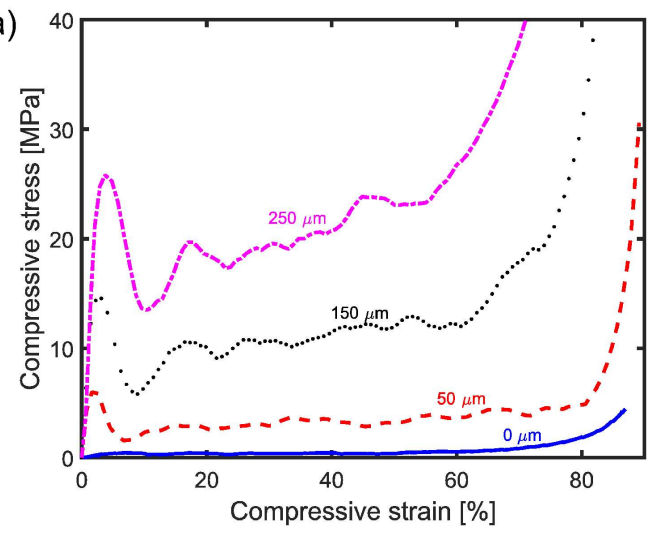

(b)

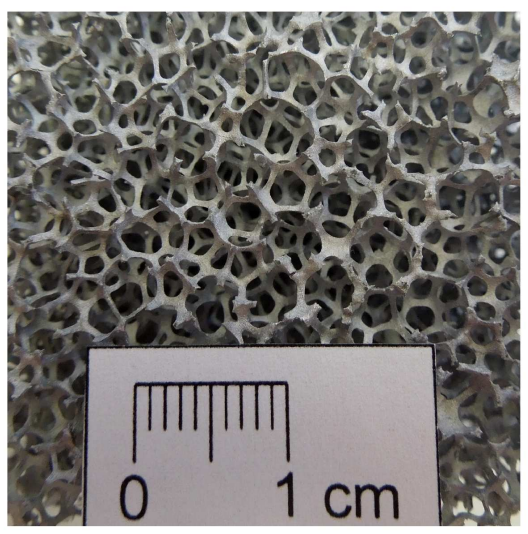

Figure 1: (a) Compressive stress-strain responses of open-cell $\mathrm{Al}$ foams (OCAFs) with different coating thicknesses. (b) Microstructure of an OCAF.

is increased by a factor of 10 compared to the uncoated OCAF. When the energy absorption capacity is normalised for the mass increase due to the coating, the relative energy absorbing capacity of the $150 \mu \mathrm{m}$ Ni-coated foam is increased by a factor of 2 compared to the uncoated OCAF $[11,12,13,15]$.

\subsection{Illustration of OCAFs as energy absorbers: frontal impact of cars}

To illustrate the capabilities of Ni-coated OCAFs as energy absorbers, frontal impacts of cars are used in this study since these may relate to a wide audience. The velocity in the frontal impact test of Euro NCAP is based on research that shows that a significant proportion of accidents takes place at $55 \mathrm{~km} / \mathrm{h}[17,18]$. In the Euro NCAP tests, cars are frontally crashed against a rigid wall with $64 \mathrm{~km} / \mathrm{h}$ which resembles two cars crashing frontally with 55 $\mathrm{km} / \mathrm{h}$ [17]. Car manufacturers calibrate the energy absorbing tubes in cars' crumple zones to this velocity. The aim of this is to make cars perform well in the Euro NCAP frontal impact test. This means that in a frontal accident at $120 \mathrm{~km} / \mathrm{h}$, for instance, only $21 \%$ of the kinetic energy is absorbed or dissipated by the crumple zone tubes, while $79 \%$ of the kinetic energy must be absorbed by other constituents of the car and the passengers.

Hence, it may be convenient to tailor the behaviour of crumple zones, and thus the behaviour of Ni-coated OCAFs when used in cars' crumple zones, that they can absorb energy at different velocities (e.g. 55 and $120 \mathrm{~km} / \mathrm{h}$ ). 
The following consideration aims to explain why this cannot be established conveniently with the regularly-coated OCAFs of which the compressive responses are shown in Fig. 1 (a).

In the following, we consider the curve denoted by ' $0 \mu \mathrm{m}$ ' in Fig. 1 (a), of an uncoated OCAF. For means of illustration only, it is assumed that crumple zone tubes made of the uncoated OCAF with the same dimensions as standard crumple zone tubes can dissipate all the kinetic energy of a car travelling at $55 \mathrm{~km} / \mathrm{h}$. As mentioned before, $79 \%$ of the car's kinetic energy is not dissipated in this case when a frontal impact takes place at $120 \mathrm{~km} / \mathrm{h}$. A number of options can ensure that all the kinetic energy is dissipated by OCAF crumple zone tubes. First, the length and/or radius of the uncoated OCAF crumple zone tubes can be enlarged. This is not a desired solution however, as it entails significantly enlarging the front of the car.

Another option is to use a Ni-coated OCAF. It is assumed here that the response denoted by ' $50 \mu \mathrm{m}$ ' in Fig. 1 (a) is high enough to dissipate the car's kinetic energy at $120 \mathrm{~km} / \mathrm{h}$. The dimensions of the OCAF crumple zone tube can hence remain the same. However, using the OCAF with the $50 \mu \mathrm{m}$ coating means that during a frontal impact at $55 \mathrm{~km} / \mathrm{h}$, compression occurs only until a strain of approximately 20\%. As a result, deceleration of the car -and the passengers- takes place significantly faster during an impact at $55 \mathrm{~km} / \mathrm{h}$ than when the uncoated OCAF is used. Consequently, passengers' bodies are exposed to large internal forces, increasing the possibility of injuries.

\subsection{Scope of this work}

A more convenient alternative would be to combine some of the responses in Fig. 1 (a) in one OCAF. This may be done by only partially coating OCAFs or gradually coating OCAFs. To the best of the authors' knowledge this cannot be incorporated by manufacturing uncoated OCAFs with functionally graded densities as the manufacturing process does not allow this. This work focuses on new hybrid OCAFs with partial Ni-coatings and with gradually increasing coatings. The aim of the work is not only to show experimentally established compressive stress-strain responses of OCAFs with partial coatings and gradually increasing coating thicknesses, but also to experimentally and numerically expose the general mechanics that govern their compressive behaviour. Another aim is to show in general lines how these foams can be employed to form passively controllable energy absorbers. For the latter aim, cars' crumple zones function as an exemplary illustration, but many other applications can be imagined. At the moment, OCAFs can actually 
not absorb the same amount of energy as crumple zone tubes. OCAFs do show qualitatively the same compressive stress-strain responses as crumple zone tubes, including the plastic collapse stress [19].

The outline of this contribution is as follows. First, materials and experimental methods are explainded. Then, the general use of OCAFs as energy absorbers in cars is discussed with the focus on the limitations of OCAFs with a single coating thickness. Subsequently, the results of compression tests on partially and gradually coated OCAFS are presented and the advantages of partially coated OCAFs and OCAFs with gradually increasing coating thicknesses are discussed. Afterwards, the mechanics of a partially coated OCAF and an OCAF with a gradually increasing coating thickness are considered to give a general understanding of the mechanics. This general understanding aims to help to tailor partially coated OCAFs and OCAFs with gradually increasing coating thicknesses in the future. Finally, conclusions are presented.

\section{Materials and methods}

\subsection{Partially and gradually coated OCAFs}

Electrodeposition is a common technique for the coating of electrically conductive structures with metals and alloys. In this work cubic samples of open-cell Al foams (OCAFs) ( $\mathrm{AlSi}_{7} \mathrm{Mg}_{0.3}$, Celltec Materials, Dresden, Germany) with an edge length of $40 \mathrm{~mm}$ and a pore size of $10 \mathrm{ppi}$, were coated by direct current plating with nanocrystalline $\mathrm{Ni}$ with a crystallite size of $50 \pm 8 \mathrm{~nm}$. The OCAFs have an average density of $0.147 \mathrm{~g} / \mathrm{cm}^{3}$ with a standard deviation of $0.021 \mathrm{~g} / \mathrm{cm}^{3}$. The size of the pores is around $4.5 \mathrm{~mm}$ and the struts have a triangular cross-section with a triangle edge length of approximately $0.5 \mathrm{~mm}$. A commercial Ni sulfamate electrolyte (Enthone GmbH, Langenfeld, Germany) with a Ni content of $110 \mathrm{~g} / \mathrm{L} \mathrm{Ni}$ was used at a $\mathrm{pH}$ of 3.8 and a temperature of $40^{\circ} \mathrm{C}$. To obtain a qualitatively good coating, the pretreatment steps of pickling and electroless plating preventing the $\mathrm{Al}$ from dissolving in the acid $\mathrm{Ni}$ electrolyte have been performed according to the existing literature [20]. To guarantee a homogeneous coating over the cross-section, a cage-like anode filled with Ni pellets (Ampere GmbH, Dietzenbach, Germany), as described by Jung et al. [12, 13], was used.

In contrast to the state of the art in fully coating OCAFs with nickel and nickel alloys $[20,21,22]$ or with copper $[23,24,25]$, the OCAFs in this work were only partially coated. Each partially coated OCAFs sample of $40 \times 40 \times 40 \mathrm{~mm}^{3}$ was manufactured by submerging only half of the foam 


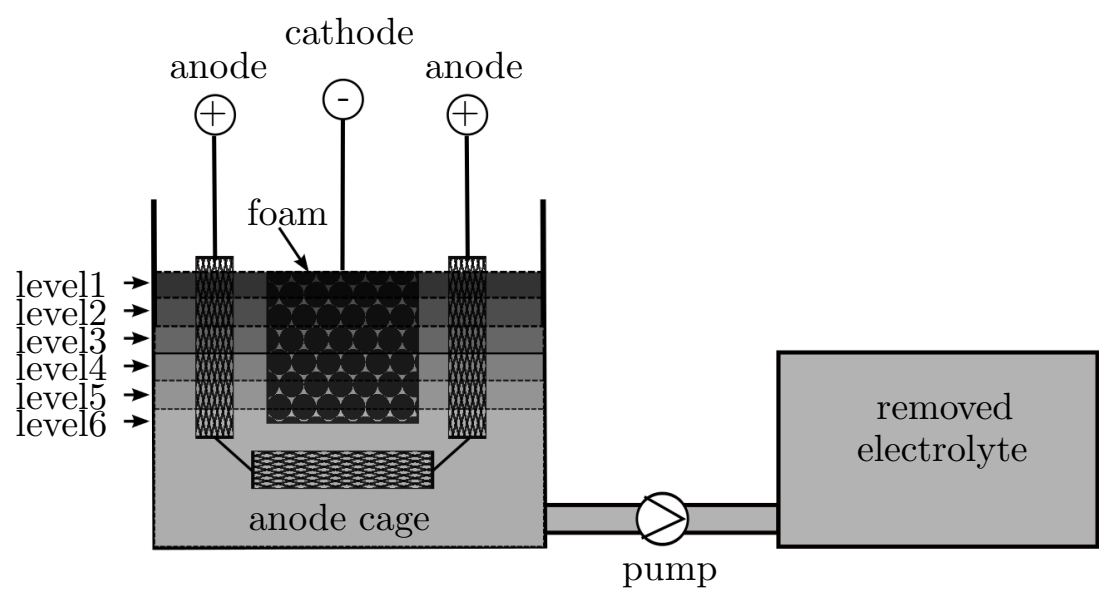

Figure 2: Schematic draving of the electrodeposition process to produce gradually coated foams. Outlined are six different electrolyte levels, which occure successively over the plating time.

in the electrolyte and coating it with $50 \mu \mathrm{m} \mathrm{Ni}$ using a current density of $1.5 \mathrm{~mA} / \mathrm{cm}^{2}$. The resulting hybrid $\mathrm{Ni} / \mathrm{Al}$ OCMF consists of an uncoated part of pure $\mathrm{Al}$ with a height of $20 \mathrm{~mm}$ and a coated part with a height of $20 \mathrm{~mm}$ and a homogeneous coating thickness of $50 \mu \mathrm{m} \mathrm{Ni}$. These foams are from here onwards referred to as partially coated OCMFs. The hybrid metal foams with a gradually increasing coating thickness from bottom to top are prepared by continuously removing the electrolyte during the coating process with a pump and lowering the electrolyte level. As a result, the surface of the foams exposed to the electrolyte reduces exponentially and the applied current density increases exponentially with the plating time. The resulting hybrid foams have an exponentially increasing coating thickness from $0 \mu \mathrm{m}$ at the bottom up to $325 \mu \mathrm{m} \mathrm{Ni}$ at the top of the foam. As a result of the increasing current density, the crystallite size of the coating decreases. The coating process in producing gradually coated metal foams is schematically outlined in Fig. 2. Six different discrete electrolyte levels are outlined. The electrolyte is removed from the plating cell and stored in a second cell.

\subsection{Quasi-static compression tests and local strain measurement using Dig- ital Image Correlation \\ Quasi-static compression tests on the OCAFs and hybrid OCAFs were performed at a strain rate of $5 \times 10^{-3} \mathrm{~s}^{-1}$ using an ElectroPlus E10000 uni-}


versal testing machine of Ltd. Instron (Pfungstadt, Germany). In order to obtain a better understanding of the local deformation mechanisms and the effects of these micro-structural deformations on the global stressstrain response, all tests have been evaluated using Digital Image Correlation (DIC). For this purpose, the ElectroPlus E10000 universal testing machine was coupled with a CCD camera Marlin 92 F-131C of Ltd. Allied Vision Technologies (Stadtroda, Germany). Images were recorded with a frequency of $1 \mathrm{~s}^{-1}$ and coupled with the measured stress-strain response.

The evaluation of local strains was done by DIC. DIC is an optical method to measure local deformations and is based on the recognition of grey-scale patterns [26]. Samples with a homogeneous color and texture require the application of a distinguishable grey-scale pattern, but this was not necessary for the foams considered in this study as the micro-structure itself is able to function as the pattern. A software algorithm recognises this pattern even in deformed states and calculates the local deformation of the sample using the displacement of each point of the pattern. In this study, the commercial software Vic2D ${ }^{T M}$ from Ltd. Correlated Solutions (Columbia, USA) is used for DIC.

\section{Results and discussion}

\subsection{OCAFs with partial and gradual coatings as passively controllable energy absorbers - Experimental results}

In Fig. 3 the results of the quasistatic compression tests are presented.. It shows the stress-strain responses (see Fig. 3 (b)) of an uncoated OCAF (solid line), of the partially coated foam (solid line with crosses), a fully coated foam with a coating thickness of $50 \mu \mathrm{m} \mathrm{Ni}$ and of the foam with the gradually increasing coating thickness (dotted line). Fig. 3 (a) schematically outlines the coating thickness $(t)$ distribution as a function of the foam length $(L)$ in the compression direction of the foams. Fig. 3 (c) shows the local strains measured by DIC of the uncoated foam (top) and the partially coated hybrid foam (bottom).

The stress-strain diagram denoted with 'partial' belongs to the OCAF with a $50 \mu \mathrm{m}$ coating in the upper part of the foam and no coating in the other part. It clearly shows the presence of two stress plateaus. Up to a strain of approximately 30\%, the uncoated OCAF and the partially coated

OCAF show a similar stress-strain response. For larger strains however, a stress increase can be observed until a second plateau stress is reached. The 

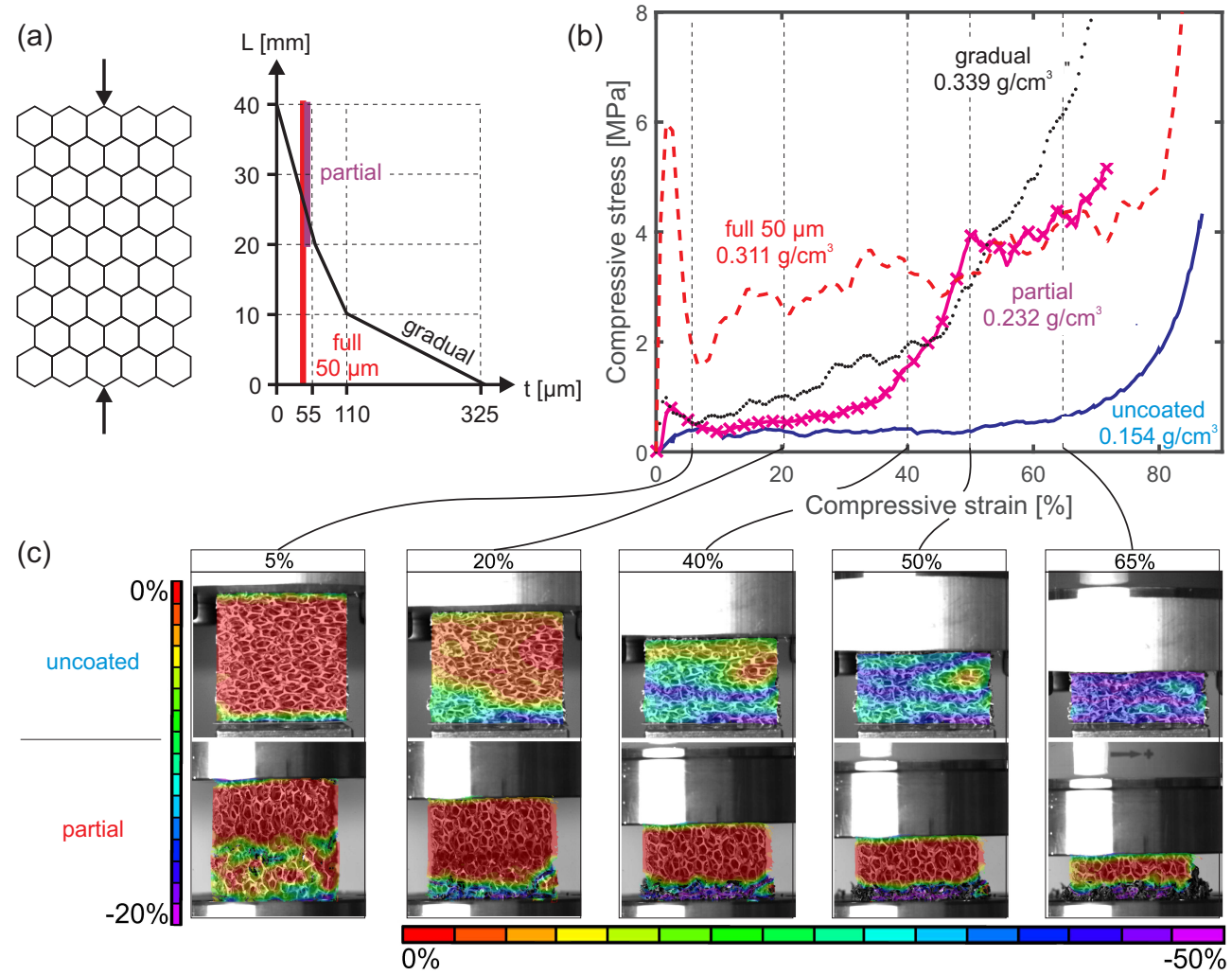

Figure 3: Schematic drawing of the coating thickness $(t)$ of the OCAF with a partial 50 $\mu \mathrm{m}$ coating and with the gradually increasing coating thickness as a function of the length in the compression direction $(L)$ (a). Compressive stress-strain responses of the uncoated OCAF (solid), the OCAF fully coated with $50 \mu \mathrm{m}$ (dashed), the partially coated OCAF (solid line with crosses) and the OCAF with the gradually increasing coating thickness (dotted) (b). The engineering stresses and strains are used in all diagrams in this contribution. Deformation history and local strains in vertical direction (compression direction) were determined using DIC of the uncoated OCAF (top row) and partially coated OCAF (bottom row) (c). Note that at a macroscopic strain of $5 \%$, a different strain scale is used than at the other macroscopic strains. 
lower plateau corresponds to the response of the uncoated OCAF part, while the second plateau represents the response of the OCAF coated with $50 \mu \mathrm{m}$ Ni. This can be deduced as a result from the DIC observations and the determination of the local strains. The uncoated OCAF shows similar local strains to the entire specimen during compression (see Fig 3 (c), upper row).

Strain localisations can be distinguished; these expose the locations of the bending and failure of struts during the different stages of compression. Initially, the partially coated foam however only shows local strains in the uncoated part (see the bottom part of the image at a 5\% strain). Buckling and failure of struts initially only occur in the uncoated domain, and when all the struts in the uncoated domain have failed, buckling and failure of struts in the coated domain occur and start at the bottom of the specimen. This is only visible to a limited extent in the images in Fig 3 (c), but clearly appears in the movies of the experiment (see supplementary data).

The buckling and failing of the uncoated struts take place during the first stress plateau up to a strain of $30 \%$. The stress increase between a strain of $30 \%$ and $50 \%$ is caused by the densification of the uncoated part. At a $50 \%$ strain, when the uncoated foam is totally compressed, a second stress plateau appears. This second plateau is approximately eight times higher than the plateau of the uncoated part. During this second plateau phase, the coated struts buckle and fail. Hence, the stress-strain response of the partially coated OCAF is a combination of the two stress-strain responses of the uncoated and coated foam, connected via a transition regime.

To summarise, initially the only significant deformation occurs in the uncoated domain and hence the stress-strain response with its pseudo-elastic region and the first stress plateau region (up to a strain of 30\%) is governed by the uncoated foam. Afterwards, a transition region between a strain of $30 \%$ and $50 \%$ appears, which is caused by the densification of the uncoated part and the pseudo-elastic deformation of the coated part. Only after a strain of $50 \%$, the stress-strain response is governed by the coated part of the partially coated hybrid foam.

As can be seen in Fig. 1, the height of the plateau stress can be customised by changing the coating thickness. By tailoring the coating thickness, and it is also possible to coat the uncoated part of the OCAF, the heights of the two stress plateaus can be adjusted so that only the dissipated kinetic energy of a car crashing at $55 \mathrm{~km} / \mathrm{h}$ equals the area under the first stress plateau, while the dissipated kinetic energy of a car crashing at $120 \mathrm{~km} / \mathrm{h}$ equals the area under both stress plateaus. In this way, a combination of 
the maximum compressive strains occurring during crashes at both velocities can be achieved, which is on average optimal for both impact velocities. This means that deceleration takes place over a relatively long period of time for both impact velocities, ensuring that the possibility of injuries is as small as possible. For the partially coated hybrid foams at an impact velocity of 55 $\mathrm{km} / \mathrm{h}$, deceleration will not be as small as in the case of the uncoated OCAF, and for an impact velocity of $120 \mathrm{~km} / \mathrm{h}$, deceleration will not be as small as in the case of the OCAF with the $50 \mu \mathrm{m}$ coating. However, by partially coating the OCAF, the deceleration will on average be as small as possible for both impact velocities.

Car accidents do however not take place at a limited number of velocities, but over a continuous range of velocities. From this perspective, an even better alternative would be not to combine a number of the responses in Fig. 1 (a) but to combine all responses between the lowest and highest response in Fig. 1 (a), also those that are not shown. An OCAF that combines all possible responses can be created by gradually changing the coating thickness. This was done for the gradually coated foam by pumping out the electrolyte leading to an exponentially increasing coating thickness. The resulting stress-strain response is shown by the dotted curve in Fig. 3 (b). Neither one nor a couple of distinct stress plateaus can be observed for this curve, but a gradually increasing stress can be seen, which combines all possible discrete stress plateaus. Obviously, the increase of this curve strongly depends on the coating thickness distribution of the OCAF. As there are no discontinuous changes in the coating thickness from one pore layer to the next, the foam cannot be divided into discrete sub-parts as is possible with the partially coated foam. The coating thickness even changes over the length of a single strut. Consequently, the bending stiffness of the struts changes not only over the length of the foam but also over the length of one pore in compression direction. As a result, there are no distinct stress plateaus visible for the gradually increasing coating thickness. The stress-strain response results from consecutive pore collapses.

At this point, it is interesting to investigate the mechanics of the partially coated OCAF and the OCAF with the gradually increasing coating during compression using numerical predictions. This will help to gain a better understanding of the deformation processes in the partial hybrid OCAF and the gradual hybrid OCAF, as it shows even more details than the experimentally obtained strain fields. 


\subsection{Simplified mechanical model for partially and gradually coated foams}

The different behaviours of the uncoated, partially coated, gradually coated and fully coated OCAFs can also be observed in numerical results. In the computations, the OCAFs are presented as a structure of eight random rectangles stacked vertically (see Fig. 4). Each strut is modelled using approximately ten non-linear beams, which are given as a linear elastic material response. Failure of the beams is not incorporated. Frictionless contact between the beams is accounted for as well as frictionless contact between the struts and two vertical walls (which are not shown in Fig. 4). In the computations in which the uncoated OCAF is represented, the diameter of all struts is $2.0 \%$ of the average strut length. In order to show the effects of the coating thickness, only the strut thickness is increased. Increasing the stiffness and yield strength of the Ni-coating in comparision to only thicker Al struts will not be investigated in this early stage of modelling. In the computations of the partially coated OCAF, the strut diameter in the domain that represents the coated part (the bottom part in Fig. 3) is changed to $2.5 \%$ of the average strut length. For the fully coated OCAF, the strut diameter of the whole foam is changed to $2.5 \%$ of the average strut length. In the computations of the OCAF with the gradually increasing coating, the OCAF is represented by a partially coated foam, where each following pore layer (rectangle) from the top to the bottom has a thicker coating (i.e. diameter) than the former pore layer, but the coating thickness is homogeneous within each pore layer. The struts of the top rectangle have a diameter of $2.0 \%$ of the average strut length, the same as in the computations of the uncoated OCAF. For each rectangle downwards, the strut diameter increases by $0.2 \%$ of the average strut length. This enables the outlined simplification to qualitatively represent a gradually coated foam. For a better comparison of the effect of the increasing strut thickness, the relative densities of the different foams are calculated in respect to the uncoated OCAF. Hence, the uncoated OCAF was normalised to a density of $\rho=1$.

The results of the computations are shown in Fig. 4. Even though the computations simplify the experiments substantially, the same qualitative trends of the experimentally established stress-strain responses can be observed in the computed responses (see Fig. 4 (a)). The difference between the mechanics of the different OCAFs can also be observed, although strut failure is not incorporated. If first the stress-strain response of the uncoated OCAF is regarded (blue curve in Fig. 4 (a)), it is clear that a number of almost discrete slope changes are present. Each slope change is caused by the 


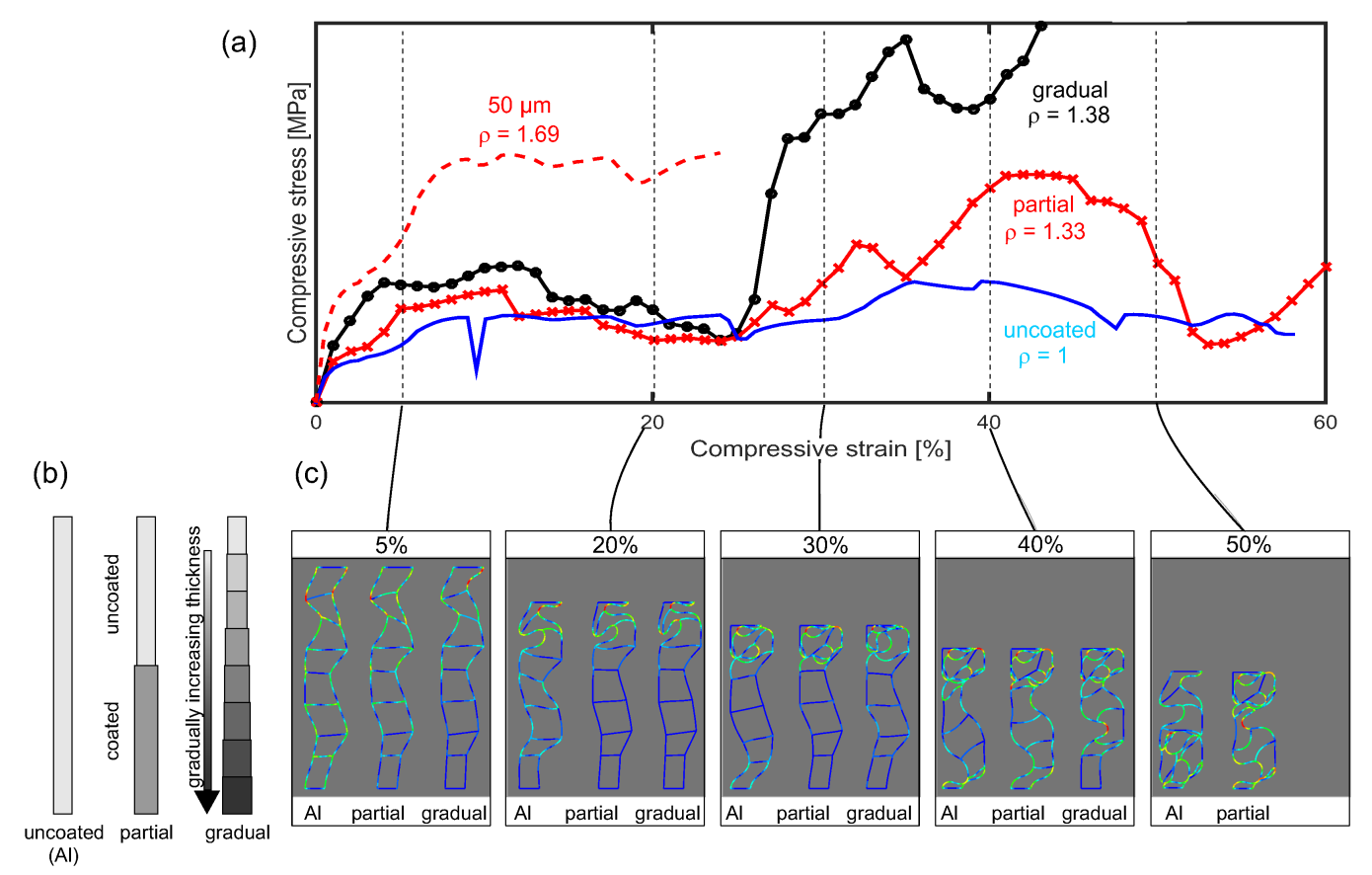

Figure 4: Compressive stress-strain responses established by simplified numerical computations. The uncoated OCAF is shown as a solid blue line, the partially coated OCAF as solid line with crosses, the fully coated OCAF as dashed red line and the OCAF with the gradually increasing coating as a solid black line with circles (a). Schematic drawing of the strut tickness distribution in the eight rectangle cells for the different types of OCAFs (b). Deformed configurations including the Von Mises stresses at different macroscopic strains of the uncoated OCAF (left), the partially coated OCAF (centre) and the OCAF with the gradually increasing coating (right) (c). The absolute values of the stresses and Von Mises stresses are not given. Blue Von Mises stresses are low, while red stresses are maximum. Note that the scales are different for each configuration shown. The deformed OCAF with the gradually increasing coating at a macroscopic strain of $50 \%$ is not shown as convergence was not reached.

buckling of a strut or the occurrence of a contact between two struts or a strut and a vertical wall. Since in the experiments a significantly larger number of struts is present in horizontal direction (i.e. the direction perpendicular to the compression direction), the buckling and failure of one strut does not lead to an observable slope change as is the case in the numerical results. Nevertheless, a rather distinct stress plateau can be seen in the numerically 
computed response of the structure representing the uncoated OCAF.

\subsubsection{Response of the OCAF with the partial coating}

Similar to the experimental response, the numerically computed response of the partially coated OCAF shows two stress plateaus (Fig. 4 (a)). The height of the first stress plateau is at approximately the same level as the computed stress plateau of the uncoated OCAF. The second stress plateau is not as distinct as in the experimental response, but a clear increase of the stress can be observed for strains larger than approximately 30\%. The fact that the second stress plateau is not as distinct as in the experimentally observed response is again due to the fact that only a few struts are present in the simulations.

If the computed mechanics of the uncoated and partially coated OCAF are compared (Fig. 4 (a)), it can be seen that they are similar to those observable in the experiments (Fig. 3). At a macroscopic strain of 5\%, Von Mises stresses are clearly present in the entire structure representing the uncoated OCAF, but the stresses in the structure representing the uncoated OCAF are more distinct in the top part of the domain that represents the uncoated part. If the deformations of both structures are compared for increasing macroscale strains, it can be seen that for both structures buckling starts at the top of the structure. However, buckling starts also in the lower part of the structure representing the uncoated OCAF at relatively small macroscopic strains, whereas this is not the case for the structure representing the partially coated OCAF. Buckling of struts in the coated part of the partially coated OCAF only starts when all struts in the uncoated domain have already buckled. As the struts in this coated domain have a larger diameter, larger forces are required to make them buckle, leading to a larger macroscopic stress. This corresponds to the experimental stress-strain responses and DIC images.

\subsubsection{Response of the OCAF with the gradually increasing coating}

The stress-strain response computed for the structure representing the OCAF with the gradually increasing coating also shows a significant number of slope changes. Even a decrease of the stress can be observed, which is not present in the experimentally established response. This corresponds to the plastic collapse of one rectangle. Again, these slope changes are caused by the limited number of struts present in the structure. In a real foam, neighbouring struts/pores will stabilise the struts in the simulation and hence, 
weakening is also present in the experiments, albeit less pronounced. Compared to the responses of the uncoated and partially coated OCAF however, the response increases significantly until no convergence can be reached in the computations.

If the computed mechanics are compared to those of the partially coated OCAF, certain differences can be observed. At a 5\% strain, stresses are more distinctively present in the part with thin struts (the top part in Fig. 4). At a $20 \%$ strain, the thinnest struts in the structure representing the OCAF with the gradually increasing coating have buckled more than those in the structure representing the partially coated OCAF. On the other hand, the struts in the rectangle below the top one, have buckled less. A similar difference in mechanics can be observed at a 30\% macroscopic strain. At a $40 \%$ strain, the bottom struts of the structure representing the partially coated OCAF are clearly buckling, but these struts do not buckle yet in the structure representing the OCAF with the gradually increasing coating because they have a significantly larger diameter. Hence, in the structure representing the OCAF with the gradual increasing coating, buckling starts in the thinnest struts and progresses towards slightly thicker struts. In the partially coated OCAF, buckling starts in the uncoated struts, and when they all have buckled, it progresses to the coated struts.

\section{Conclusions}

In this contribution, two new types of open-cell Al foams (OCAFs) are introduced: one with partial Ni-coatings and one with gradually increasing $\mathrm{Ni}$ coating thicknesses. The two new OCAFs have substantially more enhanced energy-absorbing capabilities than the standard homogeneous OCAFs. The advantage of the coating in order to improve the mechanical properties instead of increasing the density of a pure aluminium foam is the only marginally reduced porosity of less than $1 \%$ for the fully coated $50 \mu \mathrm{m} \mathrm{Ni} / \mathrm{Al}$ hybrid foam. A pure aluminium foam of equal density has a $7 \%$ reduced porosity and hence a smaller densification strain and shorter plateau region occurs. Homogeneous OCAFs are extremely beneficial to energy absorption at one specific impact velocity, but fail when the impact takes place at any other velocity. On the other hand, the partially coated OCAF and OCAF with a gradually increasing coating thickness have the promising capability to absorb energy at a wide range of impact velocities. As in most applications, the impact velocity is not known a priori, the new OCAFs may be 
used to improve existing energy-absorbers and they may lead to new energyabsorbing designs.

\section{Acknowledgement}

Stéphane Bordas also thanks partial funding for his time provided by the European Research Council Starting Independent Research Grant (ERC Stg grant agreement No. 279578).

\section{References}

[1] P. Chin, X. Sun, G. Roberts, J. Spivey, Preferential oxidation of carbon monoxide with iron-promoted platinum catalysts supported on metal foams, Appl. Catal. A-Gen. 302 (1) (2006) 22-31.

[2] W. Yang, S. Yang, W. Sun, G. Sun, Q. Xin, Nanostructured palladiumsilver coated nickel foam cathode for magnesium-hydrogen peroxide fuel cells, Electrochim. Acta 52 (1) (2006) 9- 14.

[3] A. Bansiddhi, T. Sargeant, S. Stupp, D. Dunand, Porous niti for bone implants: A review, Acta Biomater. 4 (4) (2008) 773-782.

[4] T. Lu, H. Stone, M. Ashby, Heat transfer in open-cell metal foams, Acta Mater. 46 (10) (1998) 3619-3635.

[5] T. Lu, F. Chen, D. He, Sound absorption of cellular metals with semiopen cells, J. Acoust. Soc. Am. 108 (4) (2000) 1697-1709.

[6] C. San Marchi, A. Mortensen, Deformation of open-cell aluminum foam, Acta Mater. 49 (19) (2001) 3959-3969.

[7] A. Evans, J. Hutchinson, M. Ashby, Multifunctionality of cellular metal systems, Prog. Mater Sci. 43 (3) (1998) 171-221.

[8] A. Evans, J. Hutchinson, M. Ashby, Cellular metals, Curr. Opin. Solid State Mater. Sci. 3 (3) (1998) 288-303.

[9] Z. Wang, H. Ma, L. Zhao, G. Yang, Studies on the dynamic compressive properties of open-cell aluminum alloy foams, Scripta Mater. 54 (1) (2006) 83-87. 
[10] F. Yi, Z. Zhu, F. Zu, S. Hu, P. Yi, Strain rate effects on the compressive property and the energy-absorbing capacity of aluminum alloy foams, Mater. Character. 47 (5) (2001) 417-422.

[11] A. Jung, E. Lach, S. Diebels, New hybrid foam materials for impact protection, Int. J. Impact Eng. 64 (2014) 30-38.

[12] A. Jung, H. Natter, R. Hempelmann, S. Diebels, M. R. Koblischka, U. Hartmann, E. Lach, Electrodeposition of Nanocrystalline Metals on Open Cell Metal Foams: Improved Mechanical Properties, ECS Trans. 25 (41) (2010) 165-172.

[13] A. Jung, H. Natter, S. Diebels, E. Lach, R. Hempelmann, Nanonickel coated aluminum foam for enhanced impact energy absorption, Adv. Eng. Mater. 13 (1-2) (2011) 23-28.

[14] J. Banhart, Aluminium foams for lighter vehicles, Int. J. Vehicle Des. 37 (2-3) (2005) 114-125.

[15] A. Jung, S. Diebels, Hybrid metal foams: experimental observations and phenomenological modelling, Tech. Mech. 34 (1) (2014) 12-22.

[16] L. Zardiackas, D. Parsell, L. Dillon, D. Mitchell, L. Nunnery, R. Poggie, Structure, metallurgy, and mechanical properties of a porous tantalum foam, J. Biomed. Mater. Res. A 58 (2) (2001) 180-187.

[17] E. NCAP, Front impact test of euro ncap explained. URL http://www . euroncap.com/tests/frontimpact.aspx

[18] C. of Automotive Safety of the University of Adelaide, Statistical results on accidents, accessed in October 2014.

URL http://casr.adelaide.edu.au/speed/results.html

[19] A. Deb, M. Mahendrakumar, C. Chavan, J. Karve, D. Blankenburg, S. Storen, Design of an aluminium-based vehicle platform for front impact safety, Int. J. Impact Eng. 30 (8-9) (2004) 1055-1079.

[20] A. Jung, M. R. Koblischka, E. Lach, S. Diebels, H. Natter, Hybrid Metal Foams: Mechanical Testing and Determination of Mass Flow Limitations During Electroplating, Int. J. Mater. Sci. 2 (4) (2012) 97107. 
[21] Y. Boonyongmaneerat, C. Schuh, D. Dunand, Mechanical properties of reticulated aluminum foams with electrodeposited Ni-W coatings, Scripta Mater. 59 (3) (2008) 336 - 339.

[22] B. Bouwhuis, J. McCrea, G. Palumbo, G. Hibbard, Mechanical properties of hybrid nanocrystalline metal foams, Acta Mater. 57 (14) (2009) $4046-4053$.

[23] Y. Sun, R. Burgueno, A. Vanderklok, S. Tekalur, W. Wang, I. Lee, Compressive behavior of aluminum/copper hybrid foams under high strain rate loading, Mater.Sci. Eng. A 592 (2013) 111-120.

[24] Y. Sun, R. Burgueno, W. Wang, I. Lee, Modeling and simulation of the quasi-static compressive behavior of al/cu hybrid open-cell foams, Int. J. Solids and Structures 54 (0) (2015) 135 - 146.

[25] Y. Sun, R. Burgueno, W. Wang, I. Lee, Effect of annealing on the mechanical properties of nano-copper reinforced open-cell aluminum foams, Mater.Sci. Eng. A 613 (0) (2014) 340 - 351.

[26] M. Sutton, J. Orteu, H. Schreier, Image Correlation for Shape, Motion and Deformation Measurements: Basic Concepts, Theory ans Applications, Springer, Berlin, 2009. 